\title{
EDUCAÇÃO SEM DISTÂNCIAS: utilização do WebCT como ferramenta de apoio para o ensino da Terapia Intravenosa na Graduação em Enfermagem
}

Denise Costa Dias* Silvia Helena De Bortoli Cassiani**

\section{Resumo}

Esta investigação focalizou o ambiente de aprendizagem por meio da Internet, onde foi abordado o ensino da terapia intravenosa(TIV). Dentre os vários conteúdos que poderiam ser ministrados em um ambiente de aprendizagem via Internet foi escolhida a TIV pois trata-se de um tema complexo que abrange não apenas os procedimentos técnicos mas também aspectos conceituais que podem ser discutidos em um ambiente virtual de aprendizagem. Os objetivos da investigação foram desenvolver material educacional sobre Terapia Intravenosa, utilizando a Internet para orientar o aprendizado de conceitos relacionados a terapia intravenosa, proceder avaliação por especialistas do material educacional sobre Terapia Intravenosa produzido e avaliar a utilização do mesmo pelos alunos em termos de dificuldades e/ou facilidades, participação/ interação no ambiente, usabilidade das ferramentas disponíveis no ambiente. O ambiente utilizado para disponibilizar o curso na Internet foi o WebCT.

Descritores: Educação de enfermagem; Internet; Infusões Endovenosas; Injeções Endovenosas

\section{Abstract}

This investigation focused on a learning environment via Internet, through which Intravenous Thereapy (IVT) was taught. Due to its complexity, Intravenous Therapy was chosen against numerous subjects to be taught through an e-learning environment, by comprising both technical procedures and conceptual aspects that can be discussed through a virtual learning environment. The objectives of this study were to develop educational material about Intravenous Therapy to guide students through the learning related to intravenous therapy, to have the related educational material evaluated by experts, and to evaluate the students' use of this material, considering difficulties and/or advantages, participation/interaction in this environment, and usability of its tools. The interface used for the Internet-based training program was WebCT.

Descriptors: nursing education; Internet; intravenous infusion; intravenous injection Title: Distance education: use of WebCT as a support tool for teaching Intravenous Therapy in Nursing Undergraduate Programs

\section{Resumen}

La investigación focaliza el ambiente de aprendizaje por medio de la Internet, para enseñar la terapia intravenosa(TIV). Entre los distintos contenidos que se podrían ministrar vía Internet se escogió la TIV, pues se trata de un tema complejo porque abarca los procedimientos técnicos, pero también aspectos conceptuales que se pueden discutir en un aprendizaje virtual. Los objetivos consistieron en desarrollar un material educativo sobre Terapia Intravenosa, utilizando Internet para orientar el aprendizaje de los conceptos de dicha terapia, para proceder a la evaluación del material educativo producido sobre Terapia Intravenosa y evaluar el uso del mismo por los alumnos en términos de dificultades y facilidades, participación/ interacción en el ambiente, uso de las herramientas disponibles en el ambiente.

Descriptores: Educación de Enfermería; Internet; Aplicaciones Endovenosas; Inyecciones Endovenosas

Título: Educación sin distancias: uso de la WebCT como apoyo a la enseñanza de la Terapia Intravenosa en la Graduación en Enfermería

\section{Introdução}

O cenário educacional contemporâneo mostra uma forte tendência à crescente inserção dos métodos e tecnologias da Educação a Distância em um sistema integrado, permitindo o estabelecimento de cursos com combinação variável de recursos de ensino-aprendizagem, presenciais ou não presenciais.

Consideramos importante esclarecer o conceito que intitula nossa investigação, ou seja, Educação sem distâncias. Este título pode parecer inicialmente um paradoxo, mas o próprio Ministério de Educação e Cultura já reconhece que o conceito de Educação a Distância - EAD ganha uma dimensão renovada, tornando-se na verdade, uma educação sem distância.

A denominação Educação a Distância começa a ficar ultrapassada. É ressaltado que na educação, a distância, além de relativa pode ser vista sob diferentes enfoques. O que realmente importa é a sensação de distância percebida pelo aprendiz ${ }^{(1)}$.

Com a telemática, surge uma nova fase de sistemas de $E A D$, caracterizando-se como sistemas interativos abertos que utilizam recursos das telecomunicações e da informática, e podem fazer uso de mensagens de texto, áudio e vídeo.

A educação em um ambiente de rede (on-line) ocorre num outro tempo e num outro espaço. O espaço é chamado de virtual e o tempo é de natureza diversa, permitindo encontros síncronos e assíncronos, possibilitando que o processo de ensino-aprendizagem ocorra em diferentes locais e em diferentes momentos, de acordo com a conveniência de alunos e professores ${ }^{(2)}$.

No espaço virtual o ponto de articulação entre professores e alunos não é mais a sala de aula e o tempo determinado para as aulas. É preciso aprender a transitar nesse ambiente digital de aprendizagem, que é puramente relacional.

Acredita-se que esse espaço oferece soluções aos problemas de interatividade e individualização que ocorriam em outras modalidades de EAD. A Internet possibilita que os alunos, não só acessem ao conteúdo, mas também permite que estes se comuniquem com os colegas e com o instrutor.

A informática aplicada especificamente no setor da saúde é fato consumado, tanto internacional, como nacionalmente, e encontra-se em processo de acelerado desenvolvimento. Acompanhar esse crescimento vertiginoso requer compromisso com o desenvolvimento profissional( ${ }^{(3)}$.

Assim, propiciar a educação de enfermagem por meio de um ambiente digital vem ocupar um espaço importante, contribuindo com o preparo dos alunos para que estes adquiram um perfil compatível com a cultura tecnológica, para que possam contribuir em ambientes informatizados na assistência, no ensino e na pesquisa.

Como qualquer outra proposta educativa, a EAD tem diferentes perspectivas de ação filosófica, onde princípios e valores estão postos em jogo. Conforme a perspectiva de ação

\footnotetext{
* Professora doutora da Universidade Estadual do Oeste do Paraná.

** Professora Associada DEGE-EERP- USP. Escola de Enfemagem de Ribeirão Preto- Universidade de São Paulo.

E-mail do autor: denisedias@brturbo.com
} 
pode-se cair no extremo da massificação, onde a tecnologia é usada apenas para transmitir informações ou no extremo da individualização. Porém, coloca-se uma terceira possibilidade, que se estabelece pela individualização voltada para a integração e cooperação social. Aqui se insere o conceito de aprendizagem colaborativa, considerado fundamental para o desenvolvimento da educação em ambientes de rede.

A noção de aprendizagem colaborativa é de que a aquisição de conhecimentos não é um processo inerentemente individual, mas resulta de interação grupal e baseia-se nas seguintes premissas:

- cada participante tem conhecimentos e experiências individuais para oferecer e compartilhar com o grupo;

- $\quad$ quando trabalham juntos como um time, um membro ajuda o outro a aprender;

- a compreensão é ampliada por meio da exposição a diferentes pontos de vista ${ }^{(4,5)}$.

A EAD na enfermagem já tem sido bastante utilizada em outros países com resultados satisfatórios ${ }^{(6-12)}$. No entanto, no Brasil ainda são incipientes as atividades relacionadas a essa modalidade educacional e, portanto, ressaltamos a necessidade de investimentos em pesquisa nessa área.

Este estudo justifica-se na medida em que a educação de enfermagem poderá beneficiar-se amplamente dessa nova tecnologia de ensino. Dentre os vários conteúdos que podem ser ministrados por meio da Internet, foi escolhido o tema Terapia Intravenosa(TIV) por se tratar de assunto complexo, abrangendo não apenas técnicas que devem ser aprendidas em ambientes de laboratório e em situações reais, mas também vários aspectos conceituais que podem ser discutidos em um ambiente digital de aprendizagem.

É reconhecido que novas tecnologias devam ser empregadas na educação de enfermagem, trazendo potenciais benefícios. Para tanto, existe a necessidade de ajudar alunos e professores a construírem uma relação sadia com essas tecnologias, pois ao mesmo tempo que trazem grandes benefícios, acrescentam muita complexidade ao processo.

O objetivo de um ambiente digital de aprendizagem, por meio da Internet, é apoiar a comunicação, a colaboração e a coordenação das atividades de um grupo. O grande desafio reside em conseguir comunicar e aproximar as pessoas utilizando apenas ferramentas que, embora não propiciem o contato físico, ou face-a-face direto, não eliminam fatores como a afetividade na interação e podem propiciar contato virtual, que algumas vezes poderão ser mais próximos do que contatos presenciais $^{(12)}$.

Novas tecnologias educacionais, sendo novidade, requerem uma adaptação em termos operacionais, neste sentido são fornecidas orientações ${ }^{(5)}$ para a implementação do ambiente digital de aprendizagem:

1. Identificação das necessidades: o primeiro passo é identificar atividades educacionais que podem beneficiar-se do ambiente on-line e determinar que tipo de abordagem será utilizada;

2. Garantir a acessibilidade: Recursos apropriados de hardware e software precisam ser considerados;

3. Apoio administrativo: os recursos necessários para a implementação devem ser obtidos por meio da instituição de ensino ou por financiamento de agências de fomento;

4. Planejamento do curso: devem ser definidos os objetivos educacionais em termos de conteúdo, conhecimentos e habilidades a serem adquiridos;

5. Capacitação do docente: para familiarização com o ambiente com as ferramentas disponíveis;

6. Orientação aos alunos: para que estes compreendam os princípios da aprendizagem colaborativa;

7. Avaliação: especialmente em projetos pioneiros precisa medir não apenas os resultados, mas sim o contexto ambiental e as possibilidades de melhoria da educação nesse ambiente.

Como qualquer ferramenta, a Internet oferece vantagens e desvantagens, o quadro abaixo mostra uma compilação destas $^{(13)}$.

\begin{tabular}{|l|l|}
\hline \multicolumn{1}{|c|}{ VANTAGENS } & \multicolumn{1}{|c|}{ DESVANTAGENS } \\
\hline - aumento da participação ativa & - perda das dicas não- \\
pelos alunos; & verbais de \\
- tendência diminuída para domínio & comunicação; \\
do discurso ou interrupções & - sobrecarga de \\
freqüentes; & informações; \\
- tendência diminuída para & dependência da \\
realização de julgamentos de & iniciativa dos alunos; \\
acordo com aparências ou & - exigência de \\
percepções baseadas em status; & habilidades \\
- maior acesso ao professor e aos & computacionais \\
materiais do curso (24horas/7 dias & mínimas; \\
da semana); & - exigência de acesso a \\
- aumento de tempo para reflexão e & Internet (hardware e \\
síntese em comparação com & software) \\
respostas orais imediatas; & \\
- resolução colaborativa de & \\
problemas; & \\
- registro por escrito da participação & \\
dos alunos e do conteúdo & \\
discutido. & \\
\hline
\end{tabular}

Fonte: Vandervussel L, Hanson L ${ }^{(13)}$.

Quadro 1 - Vantagens e desvantagens da educação no ambiente digital.

Para abordarmos a utilização da Internet, e mais especificamente, do ambiente WebCT, para disponibilizar conteúdos educacionais, optamos pela teoria da atividade. Esta teoria diz respeito a relação homem-mundo enquanto construída historicamente e mediada por instrumentos.

\section{Estrutura básica de uma atividade}

$$
\text { Ferramenta }
$$

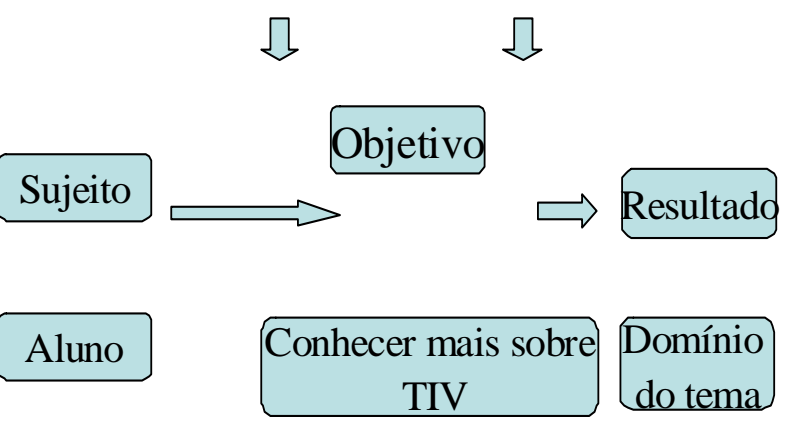

Fonte: $\operatorname{Dias}^{(14)}$.

Figura 1 - Estrutura básica de uma atividade.

As atividades humanas são consideradas como formas de relação do homem com o mundo, dirigidas por motivos, por fins a serem alcançados. A idéia de atividade envolve a noção de que o homem orienta-se por objetivos, agindo de forma intencional, por meio de ações planejadas ${ }^{(15)}$. 
Os objetivos deste estudo foram desenvolver material educacional sobre Terapia Intravenosa e disponibilizar na Internet, dentro do ambiente WebCT. Avaliar o material educacional desenvolvido envolvendo especialistas no ensino de enfermagem. E avaliar a utilização do material pelos alunos em termos de dificuldades e/ou facilidades, das ferramentas disponíveis no ambiente WebCT e a participação/interação.

\section{Metodologia}

Optamos por um estudo descritivo, desenvolvido em duas etapas, a saber:

etapa 1: Construção dos módulos do curso sobre TIV, avaliação preliminar e disponibilização no ambiente WebCT.

etapa 2: Avaliação formativa e final pelos alunos que participaram do curso on-line sobre TIV.

O estudo foi desenvolvido em escolas e cursos de graduação de enfermagem de três localidades: Escola de Enfermagem de Ribeirão Preto da Universidade de São Paulo - USP, Faculdade de Enfermagem do Centro Educacional Barão de Mauá, de Ribeirão Preto - SP; Escola de Enfermagem da Universidade Federal do Rio Grande do Sul, de Porto Alegre RS; e cursos de enfermagem da Universidade Estadual do Oeste do Paraná - UNIOESTE e da Universidade Paranaense UNIPAR, ambas de Cascavel - PR.

Na etapa 1, a população foi constituída por 4 enfermeiros, professores do ensino superior, mestres em enfermagem, que voluntariamente avaliaram o conteúdo dos módulos.

Ainda nessa etapa contamos com 15 alunos do curso de enfermagem da Universidade Estadual do Oeste do Paraná - UNIOESTE que participaram da versão piloto do curso on-line sobre TIV e o avaliaram.

$\mathrm{Na}$ etapa 2, a população foi constituída por alunos de graduação em enfermagem, preferencialmente do 5- semestre em diante, pois era importante que já tivessem alguma exposição ao conteúdo sobre terapia intravenosa para que pudessem participar discutindo suas experiências e dificuldades relacionadas ao tema. Fizeram parte da amostra 25 alunos que completaram até $50 \%$ das atividades do curso, de acordo com critérios de inclusão estipulados.

O WebCT é um ambiente para a criação, aplicação e administração de cursos baseados na Internet, desenvolvido originalmente na Universidade de British Columbia, Vancouver, Canadá, em 1995, com o objetivo de atender as necessidade do mercado acadêmico. Este software (WEBCT- Web Course Tools) auxilia o docente na tarefa de disponibilizar o conteúdo de seu curso na Internet, sem que este necessite aprender html (hiper text protocol = linguagem computacional utilizada para formatação de textos para serem veiculados na Internet) ou outras habilidades computacionais mais complexas.

O projeto de pesquisa foi submetido a análise da Comissão de Ética em Pesquisa da EERP-USP e considerado aprovado em 17 de abril de 2002, após o que foi iniciada a coleta de dados. Os alunos participantes foram orientados sobre os objetivos da pesquisa e consentiram em participar assinando termo de consentimento informado.

\section{Resultados e discussão}

\subsection{Resultados da Etapa 1}

Inicialmente foi realizado levantamento bibliográfico relacionado ao tema e o desenvolvimento do conteúdo foi organizado em módulos, em seqüência lógica, com definição dos objetivos para o aprendizado de cada módulo. Na elaboração de cada módulo tentou-se incluir atividades que demandaram a participação ativa do aluno.

A avaliação preliminar destes módulos foi realizada por especialistas no ensino de enfermagem, que, de maneira geral, consideraram o conteúdo abrangente, pertinente, organizado, obedecendo a uma seqüência lógica, claro, com objetivos instrucionais adequados e exercícios que respondiam a esses, atualizado e com conceitos corretos.

Ao disponibilizarmos os módulos do curso no ambiente WebCT realizamos um teste piloto com alunos de graduação em enfermagem. Estes alunos, de maneira geral, concordaram com a apropriação da apresentação do conteúdo em termos de texto e ilustrações, clareza dos comandos do programa (conexão e ícones), interatividade do material, adequação do relacionamento interpessoal entre os alunos e entre estes e a instrutora, e relataram que sentiram-se estimulados pela novidade. Esta experiência propiciou, sobretudo, experiência para a docente sobre a utilização das ferramentas do ambiente.

\subsection{Resultados da etapa 2}

Os alunos que completaram o curso, de maneira geral, avaliaram a oportunidade de aprendizagem no ambiente online positivamente, como vemos abaixo na avaliação de uma aluna:

De primeira mão, posso dizer que adorei a idéia do curso on-line! Foi muito tranqüilo para mim, li aos poucos, voltei quando precisei, acessei os sites sugeridos e respondi às perguntas, tudo ao meu tempo. É bem diferente de um curso com palestras, exposições de slides e transparências em que a gente não sabe se presta atenção no palestrante ou faz anotações. Jóia demais [...] (A5)

Embora os alunos tenham valorizado a experiência, percebeu-se uma elevada evasão(49\%)durante o curso, e ao serem contatados a maioria informou que a dificuldade maior era falta de tempo, seguida de dificuldade de acesso. Outros estudos sobre a modalidade de educação via Internet constataram taxas elevadas de evasão, e foi observado que entre os fatores determinantes para a desistência as respostas revelavam a falta de tempo ${ }^{(16,17)}$. Aparentemente os participantes não anteciparam a necessidade real de tempo ou não gerenciam o tempo de estudo com autonomia.

Foi avaliada a utilização do material disponibilizado no WebCT e utilizado por alunos de graduação de enfermagem de três diferentes localidades. Estes mencionaram aspectos limitadores e aspectos facilitadores da ferramenta. Colocaram suas opiniões sobre a sua participação, assim como a de seus colegas e da instrutora, no ambiente on-line de aprendizagem.

Verificamos que entre os aspectos facilitadores, houve destaque para a flexibilidade espaço-temporal possibilitada pelo ambiente virtual, acesso a recursos técnicos, a interação positiva com a instrutora e aspectos relacionados à organização e clareza do conteúdo do curso. E entre os aspectos dificultadores destacaram-se problemas técnicos, falta de tempo por parte dos alunos e falta de interação pessoal.

Sobre os aspectos que facilitaram houve destaque para a conveniência (qualquer hora e qualquer local) desta modalidade educacional, sendo esta também ressaltada na literatura como uma das principais vantagens. Em estudo realizado anteriormente, os alunos mencionaram esta vantagem, e quanto mais distante do campus residiam, mais conveniente acharam o curso via Internet ${ }^{(18)}$.

Ao avaliarmos a utilização do material disponibilizado no WebCT também questionamos os alunos quanto a aspectos relacionados a utilização das ferramentas disponíveis no ambiente. E, de maneira geral, os alunos avaliaram positivamente aspectos como clareza dos comandos para conexão e ícones; interatividade do material, velocidade de carregamento das telas e a ajuda oferecida pelo programa.

Esta investigação concebeu a educação na abordagem da Teoria da Atividade onde existe a necessidade de desconstrução do paradigma tradicional de ensino, do qual os alunos e a própria instrutora/pesquisadora foram alvo ao longo de sua vida estudantil. Existe a necessidade de participação ativa tanto dos alunos, como do instrutor que deve atuar como 
mediador, com o intuito de viabilizar a aprendizagem.

\section{Considerações finais}

Acreditamos que, ao oferecer cursos baseados na Internet ou apoiados pela Internet, na educação de enfermagem, estaremos propiciando aos alunos uma familiarização maior com tecnologias de informação atuais. No entanto, não podemos utilizar essas novas tecnologias de forma ingênua e precisamos buscar espaço dentro da enfermagem para reflexões críticas sobre essas. Para que esta geração não assista à simples substituição do ensino por correspondência pelo ensino pela "telinha", nem a substituição do quadro-negro pelo monitor. Cabe a todos os envolvidos com a EAD buscar formas que possam com certeza significar o "E" como "Educação", comprometida com a aprendizagem e a formação do aluno e não simplesmente como "ensino", mera transmissão de informações ${ }^{(19)}$.

Vislumbramos que a Internet pode somar-se a outras estratégias de ensino, funcionando como instrumento de auxílio a uma prática pedagógica diferenciada, como elemento catalisador e transformador da estratégia de educação na enfermagem, permitindo maior flexibilidade, tanto para docentes, como para discentes.

Os achados que emergem desta investigação despertam reflexões que consideramos de valor para nortear trabalhos futuros, contribuindo com subsídios para futuros cursos na área de enfermagem, tanto na graduação, como na pós-graduação e na educação continuada.

A utilização de ambientes de aprendizagem colaborativa via Internet pode motivar o aluno já familiarizado com ambientes digitais e facilitar a inclusão digital daqueles que não tiveram oportunidades de familiarização prévia.

\section{Referências}

1. Tori R. Avaliando distâncias na educação. Revista Abed. Disponíve em: URL: <http://www.abed.org.br/publique/cgi/cgilua.exe/sys/ start.htm? sid=102\&tpl=printerview \&UserActive Template $=$ 4abed\&infoid=183>. Acessado em: 2003 abr 10

2. Azevedo W. Muito além do jardim de infância: o desafio do preparo de alunos e professores on-line. In: Anais do $6^{\circ}$ Congresso Internacional de Educação à Distância; 1999 mês dias; Rio de Janeiro, Brasil. São Paulo: ABED; 1999. Disponível em: URL: <http:// www.stprj.br/abed/99.html>. Acessado em: 2001 maio 10.

3. Évora YDM, Dalri MCB. O uso do computador como ferramenta para a implantação do processo de enfermagem. Revista Brasileira de Enfermagem, Brasília (DF) 2002 nov/dez;55(6):709-13.

4. Harassin LM. Learning networks: a field guide to teaching and learning online. Cambridge (MA): MIT; 1995.
5. Ansorge C, Cooley N. The role of education faculty in choosing webbased course management systems: an interview with Nancy Cooley. Faculty and Staff Development; 2001. Available from: URL: <http:// horizon.unc/TS/default.asp?show=article\&id=846>. Accessed at: 2001 Oct 8.

6. Carlton $\mathrm{KH}$. Design courses for the internet: a conceptual approach. Nurse Educator, Philadelphia (NY) 1998 May/June;23(3):45-50.

7. Rosenlund $\mathrm{CH}$, Damask-Bembenek B. Assessing the effectiveness of an online program. Nurse Educator, Philadelphia (NY) 1999;24(1):56.

8. American Association of Colleges of Nursing. AACN white paper: distance technology in nursing education: assessing a new frontier. Journal of Professional Nursing, Philadelphia (NY) 2000 Mar/ Apr;16(2):116-22.

9. Sery-Ble OR. Use and satisfaction with a browser-based teachingool in a surgical intensive care unit. Computers in Nursing, 2001 Mar/ Apr;19(2):82-6.

10. Sternberger $C$, Meyer L. Authoring with learning guidelines. Computers in Nursing, 2001 Mar/Apr;19(2):69-74.

11. Soon $\mathrm{KH}$, Sook KI, Jung CW, Im KM. The effects of internet-based distance learning in nursing. Computers in Nursing, $2000 \mathrm{Jan} /$ Feb;18(1):19-25.

12. Santarosa LC. Criação de ambientes de aprendizagem colaborativa. In: Anais do $10^{\circ} \mathrm{SBIE}$; 1999; Curitiba (PR), Brasil. Disponível em: URL: <http://www.pgie.ugrgs.br>. Acessado em: 2002 ago 18.

13. Vandervusse L, Hanson L. Evaluation of online discussions: faculty facilitation of active student learning. Computers in Nursing, 2000. Jul/yAug;18(4):181-8.

14. Dias DC. Educação sem distâncias: utilização do WebCT como ferramenta de apoio para o ensino da terapia intravenosa na graduação de enfermagem [tese de Doutorado em Enfermagem Fundamental]. Ribeirão Preto (SP): Escola de Enfermagem de Ribeirão Preto, Universidade de São Paulo; 2003. 160 f.

15. Oliveira MK. Vygotsky: aprendizado e desenvolvimento: um processo sócio-histórico. $2^{\mathrm{a}}$ ed. São Paulo: Scipione; 1995.110 p. il.

16. Coelho ML. A evasão nos cursos de formação continuada de professores universitários na modalidade de educação a distância via Internet. São Paulo: ABED; 2002. Disponível em: URL: <http:// www.abed.org.br>. Acessado em: 2003 fev 24.

17. Moraes RA. Aula virtual e democracia: Biblioteca Virtual de Educação a Distância. Brasilia (DF): Prossiga/CNPq; 2001. Referências adicionais: Brasil/Português. Disponível em: URL: <http:// www.prossiga.br/edistancia>. Acessado em: 2003 fev 12.

18. Billings DM, Connors HR, Skiba DJ. Benchmarking best practices in web-based nursing courses. Advances in Nursing Science, Germantown (TN) 2001;23(3):41-52.

19. Franco SRK. [Apresentação]. Informática na Educação: Teoria e Prática, Porto Alegre (RS) 2002 maio;5(1):7.

Data de recebimento: $20 / 08 / 2003$

Data de aprovação: 30/10/2003 